\title{
Crystallized Rate Regions in the Secondary Interference Channels
}

\author{
Adrian Kliks, Pawel Sroka \\ Poznan University of Technology \\ Chair of Wireless Communications, Poznan, Poland \\ Emails: \{akliks, psroka\}@et.put.poznan.pl \\ Jing Lv, Eduard A. Jorswieck \\ Technical University of Dresden, Dresden, Germany \\ Emails: \{Jing.Lv, Eduard.Jorswieck\}@tu-dresden.de \\ Ricardo Blasco-Serrano, Ragnar Thobaben \\ School of Electrical Engineering and ACCESS Linnaeus Centre \\ Royal Institute of Technology (KTH), Stockholm, Sweden \\ Emails: \{ricardo.blasco, ragnar.thobaben\}@ee.kth.se
}

\begin{abstract}
In this paper the problem of sum-rate maximization for secondary cognitive users (SU) in presence of a primary user (PU) transmission is considered. We assume that all of the users are equipped with multiple transmit/receive antennas and that the cognitive users must not induce more interference to the primary users than it is defined in the permissive policy and expressed in terms of a spectrum mask or interference temperature constraint. We employ game-theory to find the so-called correlated equilibrium that corresponds to the playing strategy definitions for all users. Actually, the Vickrey-Clarke-Groves theory has been applied and the regret-matching algorithm has been implemented to find the optimal playing strategy for both secondary users. New cost function has been proposed that allows for fast convergence of the developed solutions to the optimal or sub-optimal point. Extensive simulation results have been carried out to corroborate the correctness of the proposed approach.

Index Terms-Cognitive Radio, Interference Channel, Crystallized Rate Region, regret-matching algorithm
\end{abstract}

\section{INTRODUCTION}

During recent years the idea of cognitive radio has gained a lot of attention in various research communities. The possibility of utilizing unoccupied frequency bands (in a given time and location) seems to be a good solution to the problem of high spectrum underutilization. In such an approach the so-called secondary users (SUs) can transmit their data in white/gray spaces given that they do not exceed the allowable level of interference power (known as interference temperature) induced to the licensed primary user (PU). In general, three scenarios of coexistence between primary and secondary users are considered in the literature: interweave, underlay, and overlay. The cognitive SU will maximize its rate under the generic constraint of not disturbing the PU transmission. In this case the mitigation of interference is of great importance in order to improve the achievable data rates. It is particularly important in the situation when several secondary users are transmitting in the same geographic area, thus interfering with the same primary user. Hence, the problem of cooperation between the SUs arises since the total interference from all
SUs induced to the PU must not exceed the allowed threshold. Recently, the Multiple Input Multiple Output (MIMO) technology has become an enabler for increasing the throughput in modern communication systems, and it has been considered also as a means of cooperation and/or coexistence between the primary and the secondary users. The utilization of spatial diversity due to the application of MIMO technology opens new possibilities for spatial resources reuse [1]-[3].

Several concepts of interference mitigation have been proposed, such as the successive interference cancellation or treating interference as additive noise, which are applicable to different scenarios [4]-[6]. However, not all of these proposals can be applied in a straightforward manner in cognitive terminals. In [7] it has been shown that if one treats interference as noise, the $n$-user achievable rates region can be defined as the convex hull of $n$ hyper-surfaces. A novel strategy to represent this rate region in the $n$-dimensional space, by having only binary switching power control has been proposed in [8]. Based on this approach one can illustrate the achievable rateregion in form of a crystal with the straight lines connecting any two adjacent corner points.

Different approaches, such as utility and game theory, are considered when solving the power (or generally resource allocation) optimization problem [7]-[10]. The game-theoretical solutions attempt to find equilibria, where each player of the game adopts a strategy that (s)he is unlikely to change. The best known and commonly used equilibrium is the Nash equilibrium [11]. However, the Nash equilibrium describes only the individual payoff of each player, and it may not be efficient from a system point of view. A better performance can be achieved using the correlated equilibrium [12], in which each user considers the others behaviors to explore the mutual benefits. In order to find the correlated equilibrium one can formulate a linear programming problem and solve it using one of the known techniques, such as the Simplex Algorithm [13]. However, in case of MIMO systems the number of available game strategies is high and the linear 
programming solution becomes very complicated and costly. Thus, a distributed solution can be applied, such as the regret matching learning algorithm proposed in [8], to achieve the correlated equilibrium at lower computational cost. Moreover, recently some papers have been presented, where the so-called satisfaction equilibrium has been used instead of the Nash or Pareto equilibria. This kind of equilibrium takes into account also the quality-of-service constraints and can be summarized as follows: a given player does not change its current strategy unless (s)he is not satisfied with the obtained results [14]. In this paper we deal with the application of the crystallized rate regions in the secondary interference channel. In that case two secondary MIMO users would like to maximize their total sum-rate in a certain time and given location. However, the total interference introduced by the secondary users to the primary user cannot exceed the allowed threshold. It means that the obtained set of potential solutions has to ensure that the interference requirement will be fulfilled.

The remainder of this paper is structured as follows: Section II introduces the considered system model, and the concept of crystallized rate regions for MIMO transmission is formulated. Section III describes the application of the correlated equilibrium concept in the rate region formulation. The following Section IV outlines the mechanism design for application of the proposed concept in the 2-user secondary interference MIMO channel, including the Vickey-Clarke-Groves (VCG) auction utility formulation. Finally, Section V summarizes the simulation results obtained for the considered specific cases, and Section VI draws the conclusions.

\section{System Model And Idea of CRystallized Rate REGION}

A multi-cell, uplink secondary MIMO interference channel in the underlay cognitive radio scenario is considered in this paper. The two secondary users case (which corresponds to the two cognitive cells case as well) has been analyzed, in which each of the two SU's mobile terminals (SMT) communicates with its own secondary base station (SBS) causing interference to the neighboring cell as well as to the Primary User (PU). Each SMT has $N_{t}$ transmit antennas, each SBS has $N_{r}$ receive antennas, and $i$-th SU can transmit data with the maximum total power equal to $P_{S i, \max }$. It is also assumed that the Primary Mobile Terminal (PMT) and primary base station (PBT) have $N_{r}$ and $N_{t}$ transmit and receive antennas, respectively. Intentionally, we do not consider the situation that the PU uses any technique to suppress harmful interference. Moreover, perfect channel knowledge in all SMT's is assumed. In order to simplify the analysis, we limit our derivation to case where two SU and one PU exist.

In the considered scenario the multi-path channel $\mathbf{H} \in \mathbb{C}^{3 N_{r} \times 3 N_{t}}$ is modeled as follows:

$$
\mathbf{H}=\left(\begin{array}{lll}
\mathbf{H}_{11} & \mathbf{H}_{12} & \mathbf{H}_{1 P} \\
\mathbf{H}_{21} & \mathbf{H}_{22} & \mathbf{H}_{2 P} \\
\mathbf{H}_{P 1} & \mathbf{H}_{P 2} & \mathbf{H}_{P P}
\end{array}\right), \quad \mathbf{H}_{i, j} \in \mathbb{C}^{N_{r} \times N_{t}} .
$$

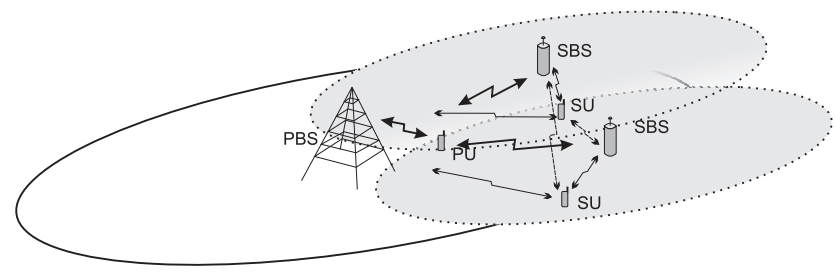

Fig. 1. Secondary MIMO Interference Channel

The channel matrix

$$
\mathbf{H}_{i, j}=\left\{h_{k, l}^{(i, j)} \in \mathbb{C}, i, j \in\{1,2, P\}, k, l \in \mathbb{N}\right\}
$$

consists of the actual values of channel coefficients $h_{k, l}^{(i, j)}$, which model the channel between transmit antenna $k$ at the $i$-th mobile terminal and the receive antenna $l$ at the $j$ th base station. In the considered two-user $2 \times 2$ MIMO secondary interference channel with one neighboring PU, six channel matrices are defined, i.e. $\mathbf{H}_{11}$ and $\mathbf{H}_{22}$ (which describe channels between the first SMT and first SBS and between the second SMT and second SBS, respectively), $\mathbf{H}_{12}$ and $\mathbf{H}_{21}$ (which describe the interference channel between the first SMT and second SBS and between the second SMT and first SBS, respectively). Furthermore, the matrices $\mathbf{H}_{1 P}$ and $\mathbf{H}_{2 P}$ denote the channels between the first and second $\mathrm{SU}$ and the PBS, the matrices $\mathbf{H}_{P 1}$ and $\mathbf{H}_{P 2}$ denote the channels between the PMT and the first and second SBS. Finally, $\mathbf{H}_{P P}$ corresponds to the channel of the primary user. Additive White Gaussian Noise (AWGN) of zero mean and variance $\sigma^{2}$ is added to the received signal.

Each user transmits the signal vector $X_{i} \in \mathbf{C}^{2}, i \in\{1,2, P\}$ through the multi-path channel. Receiver $i$ observes the desired signal, denoted as $Y_{i}$, coming from the $i$-th user. Moreover, in the interference scenario, receiver $i\left(\mathrm{SBS}_{i}\right.$ or $\left.\mathrm{PBS}_{i}\right)$ receives also interfering signals from other users located at the neighboring cell $Y_{j}, j \neq i$.

Following the analysis presented in [18], when interference is treated as noise, the achievable rates for the two-user secondary interference MIMO channel with the presence of one PU Access Point (AP) are defined as follows:

$$
\begin{gathered}
R_{1}\left(\mathbf{Q}_{1}, \mathbf{Q}_{2}\right)=\log _{2}\left(\operatorname { d e t } \left(\mathbf{I}+\mathbf{H}_{11} \mathbf{Q}_{1} \mathbf{H}_{11}^{*} .\right.\right. \\
\left.\left.\cdot\left(\sigma^{2} \mathbf{I}+\mathbf{H}_{P 1} \mathbf{Q}_{P} \mathbf{H}_{P 1}^{*}+\mathbf{H}_{21} \mathbf{Q}_{2} \mathbf{H}_{21}^{*}\right)^{-1}\right)\right) \\
R_{2}\left(\mathbf{Q}_{1}, \mathbf{Q}_{2}\right)=\log _{2}\left(\operatorname { d e t } \left(\mathbf{I}+\mathbf{H}_{22} \mathbf{Q}_{2} \mathbf{H}_{22}^{*} .\right.\right. \\
\left.\left.\cdot\left(\sigma^{2} \mathbf{I}+\mathbf{H}_{P 2} \mathbf{Q}_{P} \mathbf{H}_{P 2}^{*}+\mathbf{H}_{12} \mathbf{Q}_{1} \mathbf{H}_{12}^{*}\right)^{-1}\right)\right) .
\end{gathered}
$$

Clearly, the rate for the primary user can be expressed:

$$
\begin{aligned}
& R_{P}\left(\mathbf{Q}_{P}\right)=\log _{2}\left(\operatorname { d e t } \left(\mathbf{I}+\mathbf{H}_{P P} \mathbf{Q}_{P} \mathbf{H}_{P P}^{*} \cdot\right.\right. \\
& \left.\left.\cdot\left(\sigma^{2} \mathbf{I}+\mathbf{H}_{1 P} \mathbf{Q}_{1} \mathbf{H}_{1 P}^{*}+\mathbf{H}_{2 P} \mathbf{Q}_{2} \mathbf{H}_{2 P}^{*}\right)^{-1}\right)\right) .
\end{aligned}
$$

In the above formulas $R_{1}, R_{2}$ and $R_{P}$ denote the rate of the first and second secondary user and primary user, respectively, $\left(\mathbf{A}^{*}\right)$ denotes transpose conjugate of matrix $\mathbf{A}, \operatorname{det}(\mathbf{A})$ is the determinant of matrix $\mathbf{A}$, and $\mathbf{Q}_{i}, i \in\{1,2, P\}$ is the $i$-th user 
data covariance matrix i.e. $E\left\{X_{i} X_{i}^{*}\right\}=\mathbf{Q}_{\mathbf{i}}$ and $\operatorname{tr}\left(\mathbf{Q}_{i}\right) \leq$ $P_{i, \max }$. Let us note that the power of interference observed by the PU $\Omega=\operatorname{tr}\left\{\mathbf{H}_{1 P} \mathbf{Q}_{1} \mathbf{H}_{1 P}^{*}+\mathbf{H}_{2 P} \mathbf{Q}_{2} \mathbf{H}_{2 P}^{*}\right\}$ has to be lower than the allowable level $\xi_{P}$, i.e. $\Omega \leq \xi_{P}$. Thus, in the worst case scenario the rate of the primary user (4) will be equal to

$$
R_{P}\left(\mathbf{Q}_{P}\right)=\log _{2}\left(\operatorname{det}\left(\mathbf{I}+\mathbf{H}_{P P} \mathbf{Q}_{P} \mathbf{H}_{P P}^{*} \cdot \tilde{\sigma}^{-2}\right) .\right.
$$

where $\tilde{\sigma}^{2}$ denotes the variance of the equivalent noise (AWGN plus the interference signal). Moreover, let us define the secondary rate region as $\mathfrak{R}=\bigcup\left\{\left(R_{1}\left(\mathbf{Q}_{1}, \mathbf{Q}_{2}\right), R_{2}\left(\mathbf{Q}_{1}, \mathbf{Q}_{2}\right)\right)\right\}$. The formulas presented above allow us to calculate the rates that can be achieved by the users in the MIMO interference channel scenario in a particular case when no specific MIMO transmission technique is applied (i.e. the specific technique will be defined by proper construction of the covariance matrix $\mathbf{Q}_{i}$ ). In this paper, however, we will focus mainly on the socalled Transmit Selection Diversity (TSD) MIMO technique [16], where the SBS can decide between one of the following strategies: to put all transmit power to one antenna $\left(N_{t}\right.$ strategies, where $N_{t}$ is the number of antennas), to be silent (one strategy), or to equalize the power among all antennas (one strategy). On the other hand we will consider also the Singular Value Decomposition (SVD) MIMO approach [16], [17], denoted hereafter as SVD-MIMO. When the channel is known at the transmitter, the channel capacity can be optimized by means of some well-known MIMO transmission techniques. Precisely, one can decide for example to linearize (diagonalize) the channel by the means of Eigenvalue Decomposition (EvD) or SVD. However, let us stress that (3) has to be modified when the precoding in form of SVD method is applied. Thus, the general equations for the achievable rate computation are defined as follows:

$$
\begin{aligned}
& R_{1}\left(\mathbf{Q}_{1}, \mathbf{Q}_{2}\right)=\log _{2}\left(\operatorname { d e t } \left(\mathbf{I}+\mathbf{u}_{1}^{*} \mathbf{H}_{11} \mathbf{v}_{1} \mathbf{Q}_{1} \mathbf{v}_{1}^{*} \mathbf{H}_{11}^{*} \mathbf{u}_{1} \cdot\right.\right. \\
& \cdot\left(\sigma^{2} \mathbf{u}_{1}^{*} \mathbf{u}_{1}+\mathbf{u}_{1}^{*} \mathbf{H}_{P 1} \mathbf{Q}_{P} \mathbf{H}_{P 1}^{*} \mathbf{u}_{1}+\right. \\
& \left.\left.\left.+\mathbf{u}_{1}^{*} \mathbf{H}_{21} \mathbf{u}_{2} \mathbf{Q}_{2} \mathbf{u}_{2} \mathbf{H}_{21}^{*} \mathbf{u}_{1}\right)^{-1}\right)\right) \\
& R_{2}\left(\mathbf{Q}_{1}, \mathbf{Q}_{2}\right)=\log _{2}\left(\operatorname { d e t } \left(\mathbf{I}+\mathbf{u}_{2}^{*} \mathbf{H}_{22} \mathbf{v}_{2} \mathbf{Q}_{2} \mathbf{v}_{2}^{*} \mathbf{H}_{22}^{*} \mathbf{u}_{2} \cdot\right.\right. \\
& \cdot\left(\sigma^{2} \mathbf{u}_{2}^{*} \mathbf{u}_{2}+\mathbf{u}_{2}^{*} \mathbf{H}_{P 2} \mathbf{Q}_{P} \mathbf{H}_{P 2}^{*} \mathbf{u}_{2}+\right. \\
& \left.\left.\left.+\mathbf{u}_{2}^{*} \mathbf{H}_{12} \mathbf{u}_{1} \mathbf{Q}_{1} \mathbf{u}_{1} \mathbf{H}_{12}^{*} \mathbf{u}_{2}\right)^{-1}\right)\right)
\end{aligned}
$$

where $\mathbf{u}_{i}$ and $\mathbf{v}_{i}$ denote the set of receive and transmit beamformers, respectively, obtained for the $i$-th user. No precoding has been applied in the PMT; it has been also assumed that the PU's transmit power is equally distributed among the transmit antennas.

\section{Crystallized Rate Regions for the Secondary Interference MIMO transmission}

The idea of the Crystallized Rate Regions has been introduced in [8] and can be understood as an approximation of the achievable rate regions by a convex time-sharing hull, where the potential curves between characteristic points are replaced with the straight lines connecting these points.

Let us denote each point in the rate region as $\Phi\left(\mathbf{Q}_{1}, \mathbf{Q}_{2}\right)$. In such notation, user $i$ transmits with the total power equal to $P_{i}$, i.e. $\operatorname{tr}\left(\mathbf{Q}_{i}\right)=P_{i}$. Following the approach proposed in [8], [15] we state that instead of power control problem in finding the metrics $\mathbf{Q}_{i}$, the problem becomes finding the appropriate time-sharing coefficients of the $\left(N_{t}+2\right)^{n}-1$ corner points. For the two-user $2 \times 2$ TSD-MIMO case we will obtain 15 points, i.e. $\boldsymbol{\Theta}=\left[\theta_{k, l}\right]$ for $0 \leq k, l \leq 3 \wedge(k, l) \neq(0,0)$, which fulfill the relation $\sum_{k, l} \theta_{k, l}=1$ [15]. In our case, the time-sharing coefficients relate to the specific corner points; i.e., the coefficient $\theta_{k, l}$ defines the point, where the user one chooses the strategy $\alpha_{1}^{(k)}$ and the user two selects the strategy $\alpha_{2}^{(l)}$. Consequently, (3) can be rewritten as follows:

$$
\begin{aligned}
& R_{1}(\theta)=\sum_{k, l} \theta_{k, l} \cdot \log _{2}\left(\operatorname { d e t } \left(\mathbf{I}+\mathbf{H}_{11} \mathbf{Q}_{1}^{(k)} \mathbf{H}_{11}^{*} \cdot\right.\right. \\
& \left.\left.\cdot\left(\sigma^{2} \mathbf{I}+\mathbf{H}_{P 1} \mathbf{Q}_{P} \mathbf{H}_{P 1}^{*}+\mathbf{H}_{21} \mathbf{Q}_{2}^{(l)} \mathbf{H}_{21}^{*}\right)^{-1}\right)\right) \\
& R_{2}(\theta)=\sum_{k, l} \theta_{k, l} \cdot \log _{2}\left(\operatorname { d e t } \left(\mathbf{I}+\mathbf{H}_{22} \mathbf{Q}_{2}^{(l)} \mathbf{H}_{22}^{*} .\right.\right. \\
& \left.\left.\cdot\left(\sigma^{2} \mathbf{I}+\mathbf{H}_{P 2} \mathbf{Q}_{P} \mathbf{H}_{P 2}^{*}+\mathbf{H}_{12} \mathbf{Q}_{1}^{(k)} \mathbf{H}_{12}^{*}\right)^{-1}\right)\right),
\end{aligned}
$$

where $\mathbf{Q}_{i}^{(k)}, i \in 1,2$ denotes the $i$-th $\mathrm{SU}$ covariance matrix while choosing the strategy $\alpha_{i}^{(k)}$. Let us stress that any solution point on the crystallized rate borderline (frontier) will lie somewhere on the straight lines connecting any of the neighboring characteristic points.

Similar conclusions can be drawn for the precoded MIMO systems, where (7), which defines the achievable rate in a time-sharing approach, has to be rewritten in order to include the transmit and receive beamforming set (see (8)).

$$
\begin{aligned}
& R_{1}(\theta)=\sum_{k, l} \theta_{k, l} \cdot \log _{2}\left(\operatorname { d e t } \left(\mathbf{I}+\mathbf{u}_{1}^{*} \mathbf{H}_{11} \mathbf{v}_{1} \mathbf{Q}_{1}^{(k)} \mathbf{v}_{1}^{*} \mathbf{H}_{11}^{*} \mathbf{u}_{1} \cdot\right.\right. \\
& \cdot\left(\sigma^{2} \mathbf{u}_{1}^{*} \mathbf{u}_{1}+\mathbf{u}_{1}^{*} \mathbf{H}_{P 1} \mathbf{Q}_{P} \mathbf{H}_{P 1}^{*} \mathbf{u}_{1}+\right. \\
& \left.\left.\left.+\mathbf{u}_{1}^{*} \mathbf{H}_{21} \mathbf{v}_{2} \mathbf{Q}_{2}^{(l)} \mathbf{v}_{2}^{*} \mathbf{H}_{21}^{*} \mathbf{u}_{1}\right)^{-1}\right)\right) \\
& R_{2}(\theta)=\sum_{k, l} \theta_{k, l} \cdot \log _{2}\left(\operatorname { d e t } \left(\mathbf{I}+\mathbf{u}_{2}^{*} \mathbf{H}_{22} \mathbf{v}_{2} \mathbf{Q}_{2}^{(l)} \mathbf{v}_{2}^{*} \mathbf{H}_{22}^{*} \mathbf{u}_{2} .\right.\right. \\
& \cdot\left(\sigma^{2} \mathbf{u}_{2}^{*} \mathbf{u}_{2}+\mathbf{u}_{2}^{*} \mathbf{H}_{P 2} \mathbf{Q}_{P} \mathbf{H}_{P 2}^{*} \mathbf{u}_{2}+\right. \\
& \left.\left.\left.+\mathbf{u}_{2}^{*} \mathbf{H}_{12} \mathbf{v}_{1} \mathbf{Q}_{1}^{(k)} \mathbf{v}_{1}^{*} \mathbf{H}_{12}^{*} \mathbf{u}_{2}\right)^{-1}\right)\right)
\end{aligned}
$$

Exemplary Crystallized Rate Region for the Secondary Interference Channel

The exemplary achieved rate region and the corresponding crystallized rate regions for the $2 \times 2$ TSD-MIMO secondary interference channel is presented in Figure 2. The theoretical rate region is illustrated using the grey area, while the boundaries of the crystallized rate-region are represented as the dotted lines. One can observe that these lines connect the so-called characteristic points A, B, C, and D (together with the $(0,0)$ point $)$, creating a convex area. All of these points correspond to a specific strategy of signal transmission, for example, point A stands for the situation, when the user 1 transmits with the max allowed power during the whole transmission period, while the second secondary user is 
silent. Let us stress that the idea behind the application of the crystallized rate region is to maximize the sum-rate of the two secondary users through switching only between the characteristic points.

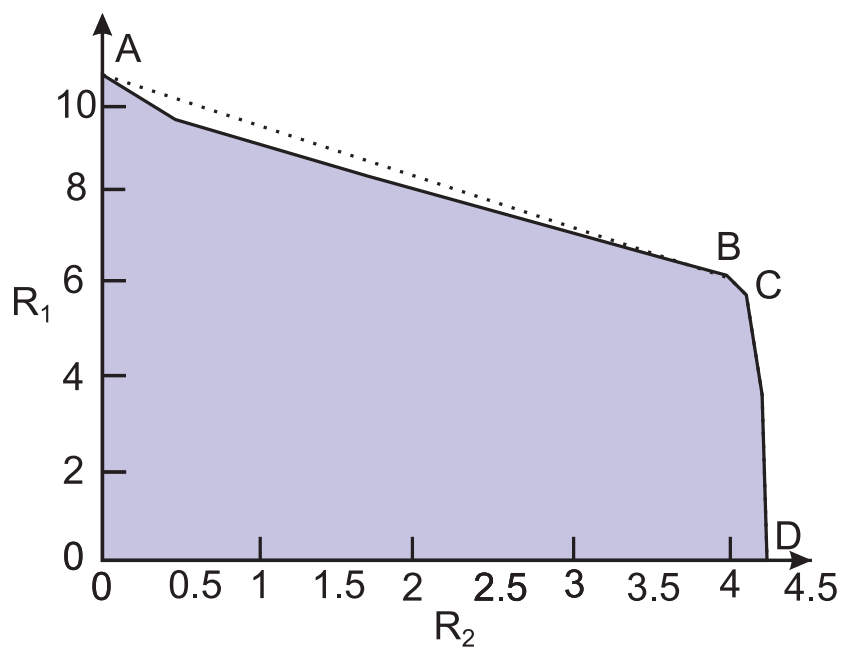

Fig. 2. Exemplary Crystallized Rate Region for the $2 \times 2$ MIMO secondary interference channel

\section{CORRElATEd EQUilibrium FOR CRYSTALLIZED INTERFERENCE MIMO CHANNEL}

In general, each user (SU) plays one of $N_{s}=N_{t}+2$ strategies $\alpha^{(k)}, 1 \leq k \leq N_{t}$. As a result of playing one of the strategies, the $i$-th SU will receive payoff, denoted hereafter $U_{i}\left(\alpha_{i}^{(k)}\right)$. The aim of each player is to maximize its payoff with or without cooperation with the other users. Such game leads to the well-known Nash equilibrium strategy $\alpha_{i}^{*}$ [20] such that

$$
U_{i}\left(\alpha_{i}^{*}, \boldsymbol{\alpha}_{-i}\right) \geq U_{i}\left(\alpha_{i}, \boldsymbol{\alpha}_{-i}\right), \forall i \in S .
$$

where $\alpha_{i}$ represents the possible strategy of the $i$-th user, whereas $\boldsymbol{\alpha}_{-i}$ defines the set of strategies chosen by the other users; i.e., $\boldsymbol{\alpha}_{-i}=\left\{\alpha_{j}\right\}, j \neq i$, and $S$ is the users set of the cardinality $n$.

However, the correlated equilibrium used in [8] instead of Nash equilibrium is defined as $\alpha_{i}^{*}$ :

$$
\begin{gathered}
\sum_{\boldsymbol{\alpha}_{-i} \in \Omega_{-i}} p\left(\alpha_{i}^{*}, \boldsymbol{\alpha}_{-i}\right)\left[U_{i}\left(\alpha_{i}^{*}, \boldsymbol{\alpha}_{-i}\right)-U_{i}\left(\alpha_{i}, \boldsymbol{\alpha}_{-i}\right)\right] \geq 0, \\
\forall \alpha_{i}, \alpha_{i}^{*} \in \Omega_{i}, \forall i \in S,
\end{gathered}
$$

where $p\left(\alpha_{i}^{*}, \boldsymbol{\alpha}_{-i}\right)$ is the probability of playing strategy $\alpha_{i}^{*}$ in a case when other users select their own strategies $\alpha_{j}, j \neq i$. $\Omega_{i}$ and $\Omega_{-i}$ (of cardinality $\left(N_{t}+2\right)^{(n-1)}$ ) denote the strategy space of user $i$ and all the users other than $i$, respectively. The probability distribution $p$ is a joint point mass function of the different combinations of users strategies.

It can be proven [8], [15] that the time-sharing coefficients $\theta_{k, l}$ are the $\left(N_{t}+2\right)^{(n-1)}$ point masses that we want to compute.

\section{Mechanism Design And Learning Algorithm}

There are two major issues that we need to cope with when optimizing the rate for the interference channel. First, to ensure the system converges to the desired point, which can be achieved by using an auction utility function. Second, a distributive solution is necessary to achieve the equilibrium. To resolve any conflicts between users, the Vickrey-ClarkeGroves (VCG) auction mechanism design is employed, which aims to maximize the utility $U_{i}, \forall i$, defined as:

$$
U_{i} \triangleq \rho R_{i}-\zeta_{i}-\delta \zeta_{i P},
$$

where $R_{i}$ is the rate of user $i$, and the fist cost term $\zeta_{i}$ is evaluated as:

$$
\zeta_{i}(\boldsymbol{\alpha})=\sum_{j \neq i} R_{j}\left(\boldsymbol{\alpha}_{-i}\right)-\sum_{j \neq i} R_{j}\left(\alpha_{i}\right) .
$$

The second cost term $\zeta_{i P}$ defines the loss of the rate of the PU, i.e.

$$
\zeta_{i P}(\boldsymbol{\alpha})=R_{P}\left(\boldsymbol{\alpha}_{-i}\right)-R_{P}\left(\alpha_{i}\right) .
$$

The binary value $\rho=\{0,1\}$ is equal to zero, when the total power of interference introduced to the PU exceeds allowed threshold $\xi_{P} . \delta$ is the scaling variable that defines the influence of the interference induced to PU on the value of the utility function. Hence, for the considered scenario with two users $i$ and $j$ the payment costs for user $i$ can be defined as in (15), where $\mathbf{Q}_{i}^{(k)}$ and $\mathbf{Q}_{j}^{(l)}$ are the covariance matrices corresponding to the strategies $\alpha_{i}^{(k)}$ and $\alpha_{j}^{(l)}$ selected by user $i$ and user $j$, respectively, which is denoted as $\alpha_{i}=\mathbf{Q}_{i}^{(k)}$. The payment $\operatorname{cost} \zeta_{j}$ follows by symmetry. Thus, the VCG utilities can be calculated using (14):

$$
\left\{U_{i}, U_{j}\right\}=\left\{U_{i}^{\prime}\left(\mathbf{Q}_{i}^{(k)}, \mathbf{Q}_{j}^{(l)}\right), U_{j}^{\prime}\left(\mathbf{Q}_{i}^{(k)}, \mathbf{Q}_{j}^{(l)}\right)\right\},
$$

where $U_{i}^{\prime}\left(\mathbf{Q}_{i}^{(k)}, \mathbf{Q}_{j}^{(l)}\right)$ is defined as in (11).

\section{Simulation Results}

First, the outage probability for both SU users as well as the total interference inducted to the primary users have been analyzed. In Figure 3 the function complementary to the outage probability is presented, while complementary cumulative distribution function of the interference introduced to the primary user is shown in Figure 4, respectively. Analyzing these results one can observe that the fairness between the users is guaranteed by the application of the proposed algorithm in the longer perspective.

In order to verify the effectiveness of the proposed solution extensive computer simulations have been carried out. The setup of the considered system is as follows: number of transmit and receive antennas of PU and SU is equal to 3 or 4 , application of the TSD-MIMO and SVD-MIMO techniques at the SU transmitter is assumed, while the transmit power of the PU is assumed to be equally distributed among all antennas. Three values of the scaling parameter are considered, i.e. $\delta \in\{0,0.5,1\}$. The maximum allowable ratio between total induced power of interferences to 


$$
\begin{aligned}
\zeta_{i} & \left(\alpha_{i}=\mathbf{Q}_{i}^{(k)}, \alpha_{j}=\mathbf{Q}_{j}^{(l)}\right)=R_{j}\left(\alpha_{i}=\mathbf{Q}_{i}^{(0)}, \alpha_{j}=\mathbf{Q}_{j}^{(l)}\right)-R_{j}\left(\alpha_{i}=\mathbf{Q}_{i}^{(k)}, \alpha_{j}=\mathbf{Q}_{j}^{(l)}\right)= \\
& =\log _{2}\left(\operatorname{det}\left(\mathbf{I}+\left(\mathbf{H}_{j j} \mathbf{Q}_{j}^{(l)} \mathbf{H}_{j j}^{*}\right) \cdot\left(\sigma^{2} \mathbf{I}+\mathbf{H}_{P j} \mathbf{Q}_{t} \mathbf{H}_{P j}^{*}\right)^{-1}\right)\right)- \\
& +\log _{2}\left(\operatorname{det}\left(\mathbf{I}+\mathbf{H}_{j j} \mathbf{Q}_{j}^{(l)} \mathbf{H}_{j j}^{*} \cdot\left(\sigma^{2} \mathbf{I}+\mathbf{H}_{P j} \mathbf{Q}_{P} \mathbf{H}_{P j}^{*}+\mathbf{H}_{i j} \mathbf{Q}_{i}^{(k)} \mathbf{H}_{i j}\right)^{-1}\right)\right), \\
\zeta_{i P} & \left(\alpha_{i}=\mathbf{Q}_{i}^{(k)}, \alpha_{j}=\mathbf{Q}_{j}^{(l)}\right)=R_{P}\left(\alpha_{i}=\mathbf{Q}_{i}^{(0)}, \alpha_{j}=\mathbf{Q}_{j}^{(l)}\right)-R_{P}\left(\alpha_{i}=\mathbf{Q}_{i}^{(k)}, \alpha_{j}=\mathbf{Q}_{j}^{(l)}\right)= \\
& =\log _{2}\left(\operatorname{det}\left(\mathbf{I}+\left(\mathbf{H}_{P P} \mathbf{Q}_{P}^{(l)} \mathbf{H}_{j j}^{*}\right) \cdot\left(\sigma^{2} \mathbf{I}+\mathbf{H}_{j P} \mathbf{Q}_{j} \mathbf{H}_{j P}^{*}\right)^{-1}\right)\right)- \\
& +\log _{2}\left(\operatorname{det}\left(\mathbf{I}+\mathbf{H}_{P P} \mathbf{Q}_{P}^{(l)} \mathbf{H}_{P P}^{*} \cdot\left(\sigma^{2} \mathbf{I}+\mathbf{H}_{j P} \mathbf{Q}_{j} \mathbf{H}_{j P}^{*}+\mathbf{H}_{i P} \mathbf{Q}_{i}^{(k)} \mathbf{H}_{i P}\right)^{-1}\right)\right),
\end{aligned}
$$

TABLE I

ACHIEVED RATES FOR BOTH SU'S, TOTAL INTERFERENCE INDUCES TO THE PRIMARY USER AND THE EXEMPLARY SELECTED STRATEGIES FOR $N_{t}=N_{r}=3$

\begin{tabular}{|c|c|c|c|c|c|}
\hline \multicolumn{7}{|c|}{ Rate of the first SU } \\
\hline$\delta$ & $\xi_{P}=0$ & $\xi_{P}=0.04$ & $\xi_{P}=0.08$ & $\xi_{P}=0.12$ & $\xi_{P}=0.16$ \\
\hline 0 & 0 & 4.988 & 9.8184 & 10.4545 & 10.4545 \\
\hline 0.5 & 0 & 5.3374 & 9.9068 & 10.4545 & 10.4545 \\
\hline 1 & 0 & 5.3436 & 7.6047 & 9.19 & 9.635 \\
\hline \multicolumn{7}{|c|}{ Rate of the second SU } \\
\hline 0 & 0 & 6.1176 & 8.3656 & 10.5373 & 10.5373 \\
\hline 0.5 & 0 & 6.2514 & 8.7324 & 10.5373 & 10.5373 \\
\hline 1 & 0 & 6.0537 & 6.3508 & 8.7254 & 8.8368 \\
\hline \multicolumn{7}{|c|}{ Total interference induced to PU } \\
\hline 0 & 0 & 0.0832 & 0.0861 & 0.1064 & 0.1064 \\
\hline 0.5 & 0 & 0.09 & 0.0992 & 0.1064 & 0.1064 \\
\hline 1 & 0 & 0.0852 & 0.0871 & 0.1042 & 0.1032 \\
\hline
\end{tabular}

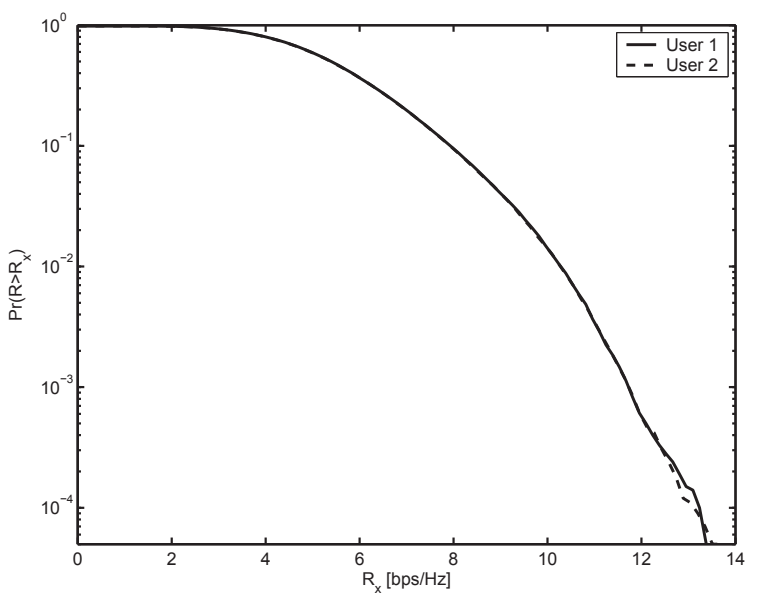

Fig. 3. Cumulative function to the outage probability for the both SU users

the PU and the total transmit power of PU are set to $\xi_{P}=0.2$.

In the case of TSD-MIMO and $N_{t}=N_{r}$ antennas the following strategies have been defined: $\alpha_{1}$ - no antenna is used for transmission; $\alpha_{i}, i=\left\{2,3, \ldots, N_{t}-1\right\}$ - total transmit power is allocated to the antenna $i$, and finally $\alpha_{N_{t}}$

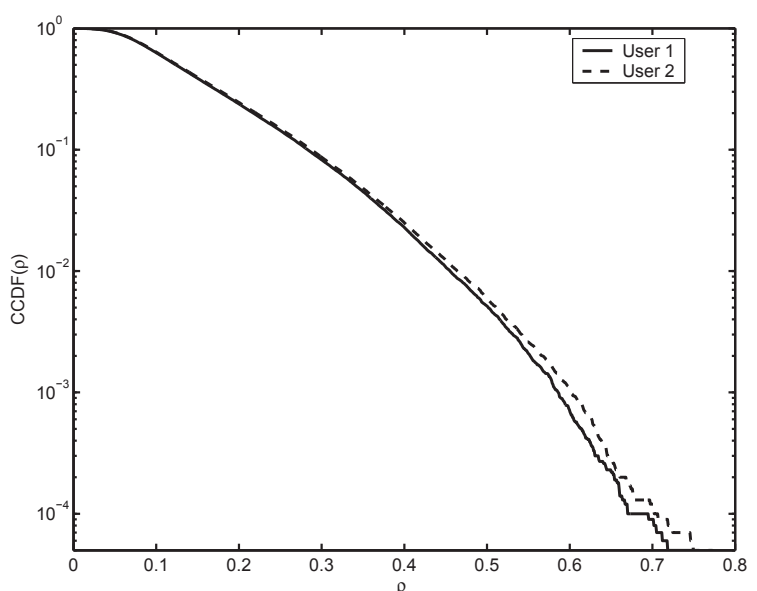

Fig. 4. Cumulative complementary distribution function of the introduced interference to the primary user.

- the maximum allowed transmit power is equally distributed among all antennas. In the second case, i.e. SVD-MIMO one, a beamforming vector is calculated that maximizes the total sum rate under the constraint of not-exceeding the allowed amount of interference induced to the primary user. Thus the strategy refers to the specific eigenvector obtained in 
TABLE II

ACHIEVED RATES FOR BOTH SU'S, TOTAL INTERFERENCE INDUCES TO THE PRIMARY USER AND THE EXEMPLARY SELECTED STRATEGIES FOR $N_{t}=N_{r}=4$

\begin{tabular}{|c|c|c|c|c|c|}
\hline \multicolumn{6}{|c|}{ Rate of the first SU } \\
\hline$\delta$ & $\xi_{P}=0$ & $\xi_{P}=0.04$ & $\xi_{P}=0.08$ & $\xi_{P}=0.12$ & $\xi_{P}=0.16$ \\
\hline 0 & 0 & 0 & 5.7498 & 10.0799 & 7.9146 \\
\hline 1 & 0 & 0 & 5.7498 & 7.3946 & 6.8695 \\
\hline \multicolumn{7}{|c|}{ Rate of the second SU } \\
\hline 0 & 0 & 0 & 7.8983 & 11.6446 & 11.4804 \\
\hline 1 & 0 & 0 & 7.8983 & 6.75 & 6.9668 \\
\hline \multicolumn{7}{|c|}{ Total interference induced to PU } \\
\hline 0 & 0 & 0 & 0.0732 & 0.1340 & 0.1299 \\
\hline 1 & 0 & 0 & 0.0732 & 0.1208 & 0.0904 \\
\hline
\end{tabular}

the SVD process. The exemplary values of achieved rates, the total interference induced to the primary user and the exemplary selected strategies for $N_{t}=3$ and $N_{t}=4$ have been presented in Tables I and II, respectively.

In the SVD-MIMO case for $\xi_{P}=0.18$ the eigenvector chosen yields the following rates for the first and second users and the total interference created to the PU: a) for $N_{t}=4$ - $R_{1}=12.098 \mathrm{~b} / \mathrm{s} / \mathrm{Hz}, R_{2}=12.21 \mathrm{~b} / \mathrm{s} / \mathrm{Hz}, \Omega=0.1676$, b) for $N_{t}=3-R_{1}=10.4545 \mathrm{~b} / \mathrm{s} / \mathrm{Hz}, R_{2}=19.53 \mathrm{~b} / \mathrm{s} / \mathrm{Hz}$ $\Omega=0.1064$.

\section{CONCLUSIONS}

In this paper the idea of crystallized rate regions has been investigated in the context of the secondary interference MIMO channel. Two approaches have been considered: TSD and SVD-MIMO. A new VCG auction utility function has been derived for the considered case and the regret-matching learning algorithm has been used to solve the formulated game. Simulation results for the selected 2-user scenario have been presented, which corroborate the results obtained applying the crystallized rates region to the current scenario. Further investigation shall focus on more precise definition of the cost function and on compact context representation; i.e., ways of effective exchange of information about the channel characteristics between the users.

\section{ACKNOWLEDGMENT}

This work was supported by the European Commission partially in the framework of FP7-ICT Network of Excellence ACROPOLIS - Advanced coexistence technologies for radio optimisation in licensed and unlicensed spectrum (contract $n$. 257626).

\section{REFERENCES}

[1] I. E. Telatar, "Capacity of multi-antenna Gaussian channels", European Transactions on Telecommunications, vol. 10, no. 6, pp. 585-595, November-December 1999

[2] R. S. Blum, "MIMO capacity with interference", IEEE Journal on Selected Areas in Communications, vol. 25, issue 5, pp. 793- 801, June 2003
[3] A. Sezgin, S. A. Jafar, H. Jafarkhani, "Optimal Use of Antennas in Interference Networks: A Tradeoff between Rate, Diversity and Interference Alignment ", accepted to IEEE GLOBECOM 2009

[4] S. Annapureddy, V. V. Veeravalli, "Sum capacity of the Gaussian interference channel in the low interference regime", in Proc. ITA Workshop, San Diego, January-February 2008

[5] X. Shang, G. Kramer, B. Chen, "A new outer bound and noisyinterference sum-rate capacity for the symmetric Gaussian interference channels", in Proc. 42nd Annual Conference on Information Sciences and Systems, CISS 2008, pp. 385-389, Princeton, 19-21 March 2008

[6] A. S. Motahari, A. K. Khandani "Capacity bounds for the Gaussian interference channel", IEEE Transactions on Information Theory, vol. 55, issue 2, pp. 620-643, February 2009

[7] M. Charafeddine, A. Sezgin, and A. Paulraj, "Rates region frontiers for $n$-user interference channel with interference as noise ", in Proc. Annual Allertone Conference on Communications, Control and Computing, Allerton, Il, September 2007

[8] M. Charafeddine, Z. Han, A. Paulraj, and J. Cioffi, "Crystallized Rate Regions of he Interference Channel via Correlated Equilibrium with Interference aas Noise "in Proc. IEEE ICC 2009, Dresden, Germany, June 14-18, 2009

[9] R. Ahlswede, "The capacity region of a channel with two senders and two receivers" in Annals of Probability, vol. 2, pp. 805-814, October 1974

[10] T. Han, K. Kobayashi "A new achievable rates region for the interference channel" in IEEE Transactions on Information Theory, vol. 27, issue 1, pp. 49-60, January 1981

[11] G. Owen, Game Theory, 3rd ed., New York: Academic, 2001

[12] R. J. Aumann, "Subjectivity and correlation in randomized strategy", Journal of Mathematical Economics, vol. 1, no. 1, pp. 67-96, 1974

[13] D. P. Bertsekas, A. Nedić, A. E. Ozdaglar "Convex Analysis and Optimization", Belmont, MA: Athena Scientific, 2003

[14] S. M. Perlaza, H. Tembine, S. Lasaulce, M. Debbah, "Quality-of-Service Provisioning in Decentralized Networks: A Satisfaction Equilibrium Approach "IEEE Journal of Selected Topics in Signal Processing, vol. PP, no. 99, pp. 1

[15] A. Kliks, P. Sroka, M. Debbah, "Crystallized Rate Regions for MIMO transmission ",Eurasip Journal on Wireless Communications and Networking, vol. 2010, 2010

[16] A. Paulraj, R. Nabar, D. Gore, "Introduction to Space-Time Wireless Communications ", Cambridge University Press, 2003

[17] G. Lebrun, J. Gao, M. Faulkner, "MIMO Transmission Over a TimeVarying Channel Using SVD", IEEE Transactions on Wireless Communications, vol. 4, no. 2, pp. 757-764, March 2005

[18] A. Goldsmith, S. A. Jafar, N. Jindal, S. Vishwanath, "Fundamental Capacity of MIMO Channels", IEEE Journal on Selected Areas in Communications, Special Issue on MIMO systems, vol. 21, 2002

[19] H. Dai, A. Molisch, H.V.Poor, "Downlink Capacity of InterferenceLimited MIMO Systems With Joint Detection ", IEEE Transactions on Wireless Communications, vol. 3, no. 2, pp. 442-453, March 2004 ", v. 8.4.0, September 2008

[20] E. G. Larsson, E. A. Jorswieck, J. Lindblom, R. Mochaourab, "Game theory and the flat-fading Gaussian interference channel", IEEE Signal Processing Magazine, vol. 26, no. 5, pp. 18-27, September 2009 